\title{
Reproductive character displacement allows two sexually deceptive orchids to coexist and attract the same specific pollinator
}

\author{
Salvatore Cozzolino ${ }^{1}$ (D) Giovanni Scopece ${ }^{1} \cdot$ Pierluigi Cortis $^{2}$. \\ Maria Rosaria Barone Lumaga ${ }^{1} \cdot$ Donata Cafasso $^{1} \cdot$ Manfred Ayasse $^{3}$
}

Received: 30 July 2021 / Accepted: 19 December 2021 / Published online: 19 February 2022

(C) The Author(s) 2022

\begin{abstract}
An increased divergence in characters between species in secondary contact can be shaped by selection against competition for a common resource (ecological character displacement, ECD) or against maladapted hybridization (reproductive character displacement, $\mathrm{RCD}$ ). These selective pressures can act between incipient species (reinforcement) or wellseparated species that already completed the speciation process, but that can still hybridize and produce maladapted hybrids. Here, we investigated two well-separated sexually deceptive orchid species that, unusually, share their specific pollinator. Sympatric individuals of these species are more divergent than allopatric ones in floral characters involved in a mechanical isolating barrier, a pattern suggestive of RCD. To experimentally test this scenario, we built an artificial sympatric population with allopatric individuals. We measured flower characters, genotyped the offspring in natural and artificial sympatry and estimated fertility of hybrids. Different from naturally sympatric individuals, allopatric individuals in artificial sympatry hybridized widely. Hybrids showed lower pollination success and seed viability than parentals. Character displacement did not affect plant pollination success. These findings suggest that RCD evolved between these species to avoid hybridization and that selection on reinforcement may be very strong even in plants with highly specialized pollination.
\end{abstract}

Keywords Ecological character displacement $\cdot$ Mechanical isolation $\cdot$ Ophrys $\cdot$ Prezygotic barriers $\cdot$ Reinforcement $\cdot$ Sexual deception

Salvatore Cozzolino

cozzolin@unina.it

1 Department of Biology, University Federico II of Naples, Naples, Italy

2 Dipartimento di Scienze della Vita e dell'Ambiente, University of Cagliari, Cagliari, Italy

3 Evolutionary Ecology and Conservation Genomics, University of Ulm, Ulm, Germany 


\section{Introduction}

Reproductive isolation can evolve between incipient species as a byproduct of niche divergence and may occur at different degrees of allopatry, parapatry or sympatry (Coyne and Orr 2004). When reproductive barriers have mostly evolved in allopatry, secondary contacts may represent a test for the speciation process, as interspecific gene flow can be completely or partly halted by previously formed reproductive barriers, or extensive hybridization and eventually genetic swamping can occur (Widmer et al. 2009; Zitari et al. 2012; Todesco et al. 2016).

If hybrids are less fit than parentals, natural selection is expected to favour local phenotypes that minimize gamete waste in interspecific crosses, and thus secondary contact zones become places where reproductive barriers may continue to evolve and reinforce, completing the speciation process (e.g., Gerhardt 1994; Noor 1995; Rundle and Schluter 1998; Servedio and Noor 2003; Haavie et al. 2004; Lemmon 2009; Sobel et al. 2010; Roda et al. 2017; Spriggs et al. 2019). The consequence of this so-called sympatric reinforcement of prezygotic isolation (Dobzhansky 1940), is an increased divergence of sympatric populations of the two incipient species (Noor 1999). Similar increased divergence can also occur in secondary contact zones between well-separated species that have already completed the speciation process, but that can still hybridize giving rise to sterile or partly sterile hybrids. In these latter cases, natural selection can decrease interspecific mating opportunities by displacing characters involved in reproduction, hence reducing the gamete waste when gene flow is already precluded by postzygotic isolation (Reproductive character displacement, RCD sensu Armbruster and Muchhala 2009). However, while sympatric reinforcement of prezygotic isolation (sensu Dobzhansky 1940) prevents gene flow that would lead to the genetic swamping of the incipient species, RCD relates to species that are already isolated to a degree sufficient to prevent their genetic amalgamation when in contact (Armbruster and Muchhala 2009).

Character divergence in different species may also result from selection to alleviate competition for a shared ecological resource, independently from their reproductive compatibility. In these cases, a high ecological similarity could preclude stable coexistence in secondary contact zones and local selection could optimize the use of the shared resource, a process that has been termed ecological character displacement (ECD; Brown and Wilson 1956; Pfennig and Pfennig 2009). This process is thus qualitatively different from reinforcement and RCD, though it generates similar patterns (e.g., Geyer and Palumbi 2003; Pfennig and Pfennig 2009) to the point that they have often been treated as a single process (e.g. van der Niet et al. 2006; Smith and Rausher 2008).

Under some circumstances, both reinforcing pressures (RCD and ECD) may occur on the same character when species come into contact secondarily. In these cases, it is difficult to determine whether the divergence is caused by competition for resources, or costly hybridization, as decreased hybridization is likely to be the pleiotropic consequence of selection for optimizing the use of a common resource in contact zones. A key example for this dual action of reinforcing selection are flowering plants and their pollinators. Competition for pollinator services (i.e., competition for a shared resource) may cause pollen limitation and lower seed sets (e.g., Caruso 2000; Bell et al. 2005) and natural selection will favour character divergence (morphological and/or phenological characters) between coflowering species that have met secondarily (i.e., ECD). However, if pre-zygotic isolation is not complete, the sharing of common pollinators may promote the transfer of heterospecific pollen and result in the formation of inviable or sterile hybrids, therefore lowering 
plant fitness (e.g., Fishman and Wyatt 1999; Norton et al. 2015; Hopkins and Rausher 2012), and selection against hybrid mating may enhance divergence in reproductive characters (i.e., RCD). Thus, competition for pollinators represents the best example of convergence between ECD and RCD as it can be considered an indirect type of RCD or ECD as pollinators represent both a form of plant reproductive isolation and a shared ecological resource for co-flowering plant species (Dayan and Simberloff 2005). Furthermore, in order to reduce the amount of maladaptive hybridization, premating isolation is less costly than postmating isolation, as it prevents the waste of gametes (Coyne and Orr 2004; Price 2007) and, because in plants, pollinator isolation is an early premating reproductive barrier, it can be predicted as one of the main targets of reinforcing selection.

Pollinator isolation reduces competition (for pollinators) between coexisting plant species (Waser 1978; Mitchell et al. 2009), and several studies have provided evidence of ECD in which this competition was the most plausible fuel (Armbruster and Herzig 1984; Stone et al. 1998; Aizen and Vázquez 2006; Muchhala and Potts 2007). Indeed, ECD is particularly common in plant species with generalized pollination where diverged lineages, once in secondary contact, often share and compete for the overlapping pollinator sets (Norton et al. 2015 and references therein). Differently, ECD involving pollinator-related characters is less common among species with distinct, specific pollinators as competition for pollinators is unlikely upon secondary contact (Beans 2014). Indeed, pollinator-specialized plant species may coexist more easily than generalist plant species (Kozak and Wiens 2006; van der Niet et al. 2006) once they are not sharing their specialized pollinator. In contrast, generalized pollinated plants can easier displace floral characters in order to avoid pollinator interspecific movements, without the extreme consequence on fitness (Hopkins and Rausher 2012) expected for pollinator-specialised plants.

Among the pollinator-specialised plant species, orchids employing sexually deceptive strategies are considered one of most extreme examples of species-specific pollination. Each Ophrys species attracts its exclusive pollinator by producing a specific floral odour bouquet. This extremely specific pollination strategy assures ethological pollinator-mediated isolation (Ayasse et al. 2011) even among closely related sympatric sexually deceptive orchids (Xu et al. 2011; Whitehead and Peakall 2014) and the fast radiation of sexually deceptive Ophrys is driven by shift in pollinator niches (Breitkopf et al. 2015; but see also Bateman et al. 2018).

Here, we examine an unusual case in which two sexually deceptive orchid species, i.e. Ophrys normanii and $O$. chestermanii, belonging to different Ophrys clades (Gögler et al. 2009), converged to the same species-specific pollinator, males of the cuckoo bumblebee Bombus vestalis, by producing the same odour bouquets and overlapping in flowering time (Gögler et al. 2015). The two species are thought to have evolved in allopatry (Gögler et al. 2009; Lussu et al. 2018), but now meet in secondary contact, to the extent that the distribution of $O$. normanii is largely overlapping with that of $O$. chestermanii. In spite of phylogenetic distance, Gögler et al. (2015) found an insignificant difference in viable seed production from intra and interspecific crosses, as often reported in this orchid genus, whose reproductive barriers largely rely on pre zygotic isolation (Scopece et al. 2007). Furthermore, hybrid seeds germinated and produced viable flowering F1 hybrids suggestive of weak, if any, early postzygotic reproductive incompatibilities. Nevertheless, it remains to be tested whether other late-acting post zygotic barriers (intrinsic, such as hybrid sterility/unviability or extrinsic, such as hybrid attractiveness to pollinators) prevent genetic admixture between $O$. normanii and $O$. chestermanii. Indeed, despite the apparent absence of premating and early post zygotic reproductive barriers, the two species maintain their genetic integrity in sympatric population, hybrids being very rare in nature (Gögler et al. 
2009). Interestingly, flower characters, as width and length of the stigmatic cavity and pollinaria length were found to be more different between sympatric than between allopatric populations of the two species (Gögler et al. 2015), a pattern suggestive of RCD (see Howard 1993). These diverging floral characters have been shown to be related to pollinator isolation in a sort of lock and key pattern shaping a mechanical barrier between the two sympatric species (Gögler et al. 2015; Lussu et al. 2019). Accordingly, sympatric individuals with floral characters impeding interspecific pollen transfer (i.e. more divergent characters) are expected to be positively selected, as opposed to allopatric individuals that experience no selection on these characters. However, if floral characters have already diverged to the point where the displaced species are no longer competing, then the signal of this reinforcing selection may also be undetectable in the contact zone. At the same time, observation of increased character divergence in sympatric populations by itself is not sufficient to demonstrate the role of these characters in species displacement as a direct link between character divergence and reproductive isolation for species pair has to be shown. Finally, character divergence may be to reduce costly hybridization (RCD) or to increase pollinator efficiency thereby reducing competition for services of the shared pollinator without shifting to a different pollinators (ECD).

To resolve these alternative hypotheses we performed a transplantation experiment by creating an artificially sympatric population where we first determined whether distributions of floral variation in our study populations are consistent across years, and consequently tested the strength of reproductive isolation between allopatric individuals and compared it with that between sympatric individuals (Martin and Harding 1981). More specifically, we addressed the following questions:

1. Is the spatial distribution of character displacement consistent with allopatric/sympatric occurrence of the two Ophrys species?

2. Are divergent floral characters correlated with pollination success (i.e., ECD)?

3. Is hybridization a cost and is it alleviated in plants with more divergent floral characters (i.e., RCD)? Is hybrid fitness lower than parental fitness?

4. Is the character divergence maintained in common garden environment and thus likely under genetic control?

5. Are allopatric individuals more likely to hybridize in artificial sympatry than natural sympatric individuals?

\section{Materials and methods}

\section{Study system and sample collection}

Ophrys normanii Wood and O. chestermanii (Wood) Gölz and Reinhard are two rare orchid species endemic to Sardinia. Ophrys normanii distribution consists of only three small populations located in the south-western portion of the island while $O$. chestermanii occurs in the same area and in two other localities along the Tyrrhenian coast (Scrugli 1990). Ophrys normanii shows a slightly different ecological preference but both species are sympatric in the locality of Perdu Carta. The two species belong to two phylogenetically distant clades, with $O$. normanii belonging to the $O$. tenthredinifera clade and $O$. chestermanii to the $O$. holoserica clade (Gögler et al. 2009; Breitkopf et al. 2015) and have clearly distinguishable flower shape (Supplementary Fig. 1). 
In 2017 we collected 25 individuals of $O$. normanii at flowering stage (to allow unambiguous identification) from an allopatric population (Fluminimaggiore) and haphazardly placed them in a small allopatric population of $O$. chestermanii of approx. 30 blooming individuals (Reigraxius, Domusnovas) (Supplementary Fig. 2) to gather a new artificial sympatric population with comparable frequencies of each species. The same source populations of $O$. normanii and $O$. chestermanii were previously investigated by Gögler et al. (2015). We then labeled all transferred $O$. normanii individuals, and $27 O$. resident chestermanii individuals in this new artificial sympatric population. As in orchids, plant phenotype is also determined by resources allocated in the tuber produced in the previous year, only in 2018 , i.e. one year after the transplantation, we collected a flower for morphological measurements from each labeled individual of $O$. normanii and $O$. chestermanii in the artificial sympatric population and in allopatric and natural sympatric populations.

At the end of the flowering season, both in 2017 and in 2018, we collected the fruits produced by each labeled individual and we also recorded pollination success in natural (Perdu Carta) and artificial (Reigraxius, Domusnovas) sympatric populations by counting the number of fruits relative to the number of flowers. At the end of the experiment, transplanted individuals were transferred back to their source population.

\section{Hybrid fitness}

To assess hybrid fitness, we used F1 hybrids between $O$. chestermanii and $O$. normanii produced from manual crosses in Gögler et al. (2015). From these crosses, three years after in vitro germination, three blooming F1 hybrid plants were obtained (two $O$. normanii $X$. chestermanii and one $O$. chestermanii $\times O$. normanii). Here, we crossed these three F1 plants (six manual crosses, each plant providing and receiving pollen from the other two) as described in Scopece et al. (2007).

In 2018, we also collected leaf material from eight labelled putative $O$. normanii $O$. chestermanii hybrid individuals identified, on morphological bases, in another newly discovered small sympatric zone (Buggerru, loc. San Niccolò) (Fig. S2). DNA was extracted and Internal Transcribed Spacers 2 (ITS2) sequences were used to confirm potential hybrid individuals (as described below). Of the eight labeled individuals, six showed parental genetic profiles (four $O$. normanii and two $O$. chestermanii) and two showed a genetic profile compatible with the F1 hybrid (i.e. presence of heterozygote sites as described in Gögler et al. 2015). From these two putative hybrids, we collected the fruits and assessed seed viability as described in Scopece et al. (2007). With this approach we couldn't distinguish the hybrid generation of the two putative hybrids (F1, F2, BCs) nor we could identify the pollen donor of their fruits.

\section{Morphological measurements}

Flowers collected from allopatric, natural sympatric and artificial sympatric populations in 2018 were photographed under a binocular microscope (Wild Heerbrugg M8) with a digital camera (Nikon Nikkor1J1) using a millimeter scale as a reference. As previously done by Gögler et al. (2015), from each flower we measured four characters: width and height of stigmatic cavity, pollinaria length, and length of outer central tepal (a flower character potentially not involved in the placement of the pollinator on the flower) (Supplementary Fig. 3). To measure pollinaria length we first removed pollinaria by using a measuring lens under the 
binocular microscope. Measurements were performed using ImageJ 1.33 software (Rasband, National Institutes of Health, USA).

\section{Seed pool paternity test}

Ripe fruits collected in natural and artificial sympatric populations in 2017 and 2018 from $O$. normanii and $O$. chestermanii individuals were harvested and stored at $4{ }^{\circ} \mathrm{C}$ until using them for molecular analysis. DNA from pooled seeds from a single fruit was extracted following Doyle and Doyle (1987). The ITS2 region was amplified by polymerase chain reaction (PCR) using primers annealing with the $3^{\prime}$ region of the $5.8 \mathrm{~S}$ and the $5^{\prime}$ region of the $25 \mathrm{~S}$ rDNA genes, by using the conditions described in Gögler et al. (2015) and purified by exonuclease I and SAP treatments (Fermentas Inc., Hannover, MD). Purified PCR products were sequenced in both directions using a modification of the Sanger dideoxy method as implemented in a double-stranded DNA cycle sequencing system with fluorescent dyes. Sequence reactions were then run on a 3130 Automated DNA Sequencer and the Sequencing Analysis software (Applied Biosystems, USA) was used to check electropherograms. Sequences were assembled with BioEdit and manually edited. ITS2 sequences showed three indels between $O$. chestermanii and $O$. normanii; hybrid fruits were thus recognized by the presence of heterozygosity at these specific sites (Gögler et al. 2015).

\section{Data analysis}

As our data were not normally distributed, we tested for differences in flower characters between $O$. normanii and $O$. chestermanii individuals in allopatry, natural sympatry and artificial sympatry using a Mann Whitney $U$ test. In the artificial sympatric population, we also tested the differences of flower characters between $O$. normanii and $O$. chestermanii hybridizing and non-hybridizing individuals. We did not test for differences in pollinaria length between hybridizing individuals, because our experimental approach does not allow the pollen donor of observed fruits to be identified. Pollination success data were compared between natural and artificial sympatry using a Mann Whitney U test. We performed a Pearson's correlation between investigated flower characters and pollination success.

Principal component analysis (PCA) of flower character involved in reproductive isolation (i.e., width and height of stigmatic cavity, pollinaria length) was also conducted as a descriptor of overall morphological differences between species in natural and artificial sympatry. We also compared floral characters of individuals from the natural and artificial sympatric populations sampled in the present study (2018) with those of individuals sampled in the same source populations by Gögler et al. (2015).

Fruit production in manual crosses between artificial F1 hybrids was compared to fruit production in intraspecific crosses reported in Gögler et al. (2015) using a Fisher's exact test. Seed viability of natural hybrids (from Buggerru, loc. San Niccolò) was compared to seeds viability in intraspecific crosses reported in Gögler et al. (2015) using a Fisher's exact test. All analyses were conducted using SPSS (v. 21.0.0.0). 


\section{Results}

Molecular analyses showed that none of the 11 fruits collected in 2017 in the natural sympatric population contained hybrid seeds, whilst 9 out of $12(75 \%)$ fruits collected in the artificial sympatric population were of hybrid origin (5 out of 7 on $O$. chestermanii and 4 out of 5 on $O$. normanii). Similarly, in 2018, molecular analyses showed 8 out of $32(25 \%)$ fruits collected in the artificial sympatric population were of hybrid origin (4 out of 23 fruits collected on $O$. chestermanii and 4 out of 9 fruits collected on $O$. normanii).

In natural sympatry, we found significant differences between $O$. normanii and $O$. chestermanii in pollinaria length $(\mathrm{U}=16.000, \mathrm{Z}=-5.530, P<0.001, \mathrm{n}=47)$ and width of the stigmatic cavity $(\mathrm{U}=91.000 ; \mathrm{Z}=-3.937 ; P<0.001, \mathrm{n}=47)$. Height of stigmatic cavity was also marginally significantly different $(\mathrm{U}=186.500 ; \mathrm{Z}=-1.904 ; P=0.057, \mathrm{n}=47$ ) (Fig. 1). Length of outer central tepal was not significantly different between $O$. normanii and $O$. chestermanii $(\mathrm{U}=224.500 ; \mathrm{Z}=-1.077 ; P=0.281, \mathrm{n}=47)$.

In the allopatric populations, we found significant differences between $O$. normanii and $O$. chestermanii in pollinaria length $(\mathrm{U}=81.000, \mathrm{Z}=-5.450, P<0.001, \mathrm{n}=67)$. All the other characters were not significantly different between the two investigated species (width of stigmatic cavity: $\mathrm{U}=469.500, \mathrm{Z}=-0.184, P=0.854, \mathrm{n}=67$; height of stigmatic cavity: $\mathrm{U}=456.500, \mathrm{Z}=-0.361, P=0.718, \mathrm{n}=67$; length of outer central tepal: $\mathrm{U}=368.500$, $\mathrm{Z}=-1.277 ; P=0.201, \mathrm{n}=66$ ) (Fig. 1).

In artificial sympatry, we found significant differences between $O$. normanii and $O$. chestermanii in pollinaria length $(\mathrm{U}=39.500, \mathrm{Z}=-5.043, P<0.001, \mathrm{n}=47)$. All the other characters were not significantly different between the two investigated species (width of stigmatic cavity: $\mathrm{U}=270.000, \mathrm{Z}=-0.792, P=0.429, \mathrm{n}=50$; height of stigmatic cavity: $\mathrm{U}=228.000, \mathrm{Z}=-1.625, \mathrm{P}=0.104, \mathrm{n}=50$; length of outer central tepal: $\mathrm{U}=307.500$, $\mathrm{Z}=-0.058 ; P=0.953, \mathrm{n}=50$ ) (Fig. 1).

Overall, floral characters of individuals of sympatric and allopatric populations of Ophrys chestermanii and $O$. normanii samples in 2018 (i.e.present study) were similar to those of individuals sampled in the same source populations by Gögler et al. (2015) (Fig. 2). In particular, Ophrys chestermanii individuals from the natural sympatric population collected by Gögler et al. (2015) and present study were not significantly different in terms of pollinaria length $(\mathrm{U}=203.000, \mathrm{Z}=-1.083, P=0.279, \mathrm{n}=45)$, height of the stigmatic cavity $(\mathrm{U}=248.000, \mathrm{Z}=-0.046, P=0.963, \mathrm{n}=45)$ or width of the stigmatic cavity $(\mathrm{U}=189.500, \mathrm{Z}=-1.395, P=0.163, \mathrm{n}=45)$. $O$. normanii individuals from the natural sympatric population collected by Gögler et al. (2015) and present study were not significantly different in terms of pollinaria length $(\mathrm{U}=199.500, \mathrm{Z}=-0.519, P=0.604, \mathrm{n}=42)$, height of the stigmatic cavity $(\mathrm{U}=181.500, \mathrm{Z}=-0.982, P=0.326, \mathrm{n}=42)$ or width of the stigmatic cavity $(\mathrm{U}=148.500, \mathrm{Z}=-1.810, P=0.070, \mathrm{n}=42)$.

PCA showed a larger separation between $O$. chestermanii and $O$. normanii in natural sympatry than in artificial sympatry (Fig. 3). In the artificial sympatric population, floral characters of hybridizing individuals of $O$. chestermanii and $O$. normanii were not significantly different $(\mathrm{N}=8$; width of stigmatic cavity: $\mathrm{U}=7.000, \mathrm{Z}=-0.289$, $P=0.886$; height of stigmatic cavity: $\mathrm{U}=4.500, \mathrm{Z}=-1.084, P=0.343$; length of outer central tepal: $\mathrm{U}=8.000, \mathrm{Z}=0.000 ; P=1)$. Non-hybridizing individuals of $O$. chestermanii and $O$. normanii showed no significant differences in terms of height of stigmatic cavity $(\mathrm{N}=39 ; \mathrm{U}=139.000, \mathrm{Z}=-1.373 ; P=0.181)$, or in terms of width of stigmatic cavity $(\mathrm{N}=39 ; \mathrm{U}=156.000, \mathrm{Z}=-0.883 ; P=0.392)$. Hybridizing and non-hybridizing individuals of $O$. chestermanii were not significantly different in terms of height of 

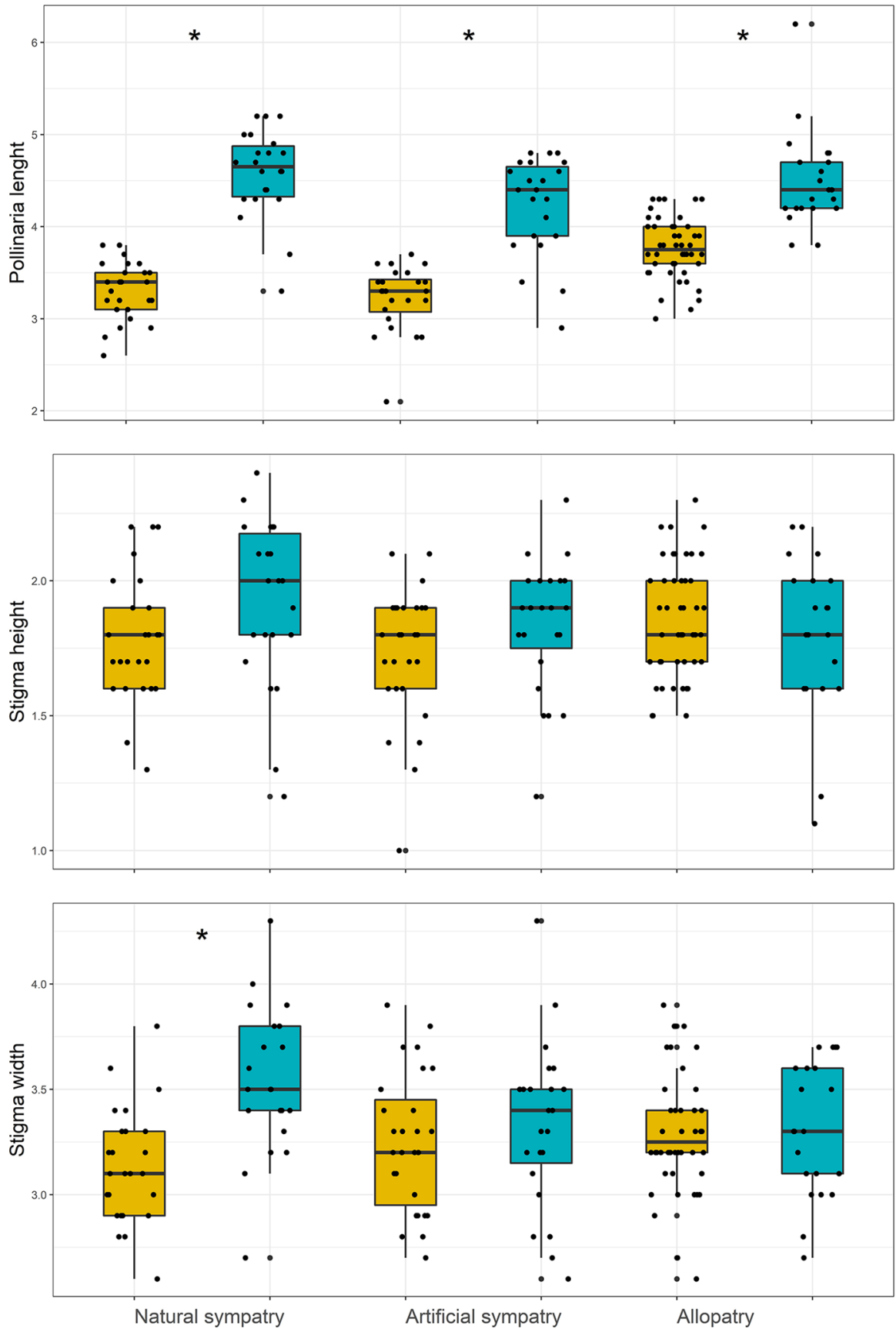

Fig. 1 Comparison of pollinaria length, stigma height and stigma width of Ophrys normanii (blue) and Ophrys chestermanii (yellow) among allopatric, natural and artificial sympatric populations sampled in 2018. Significant differences are indicated by stars 

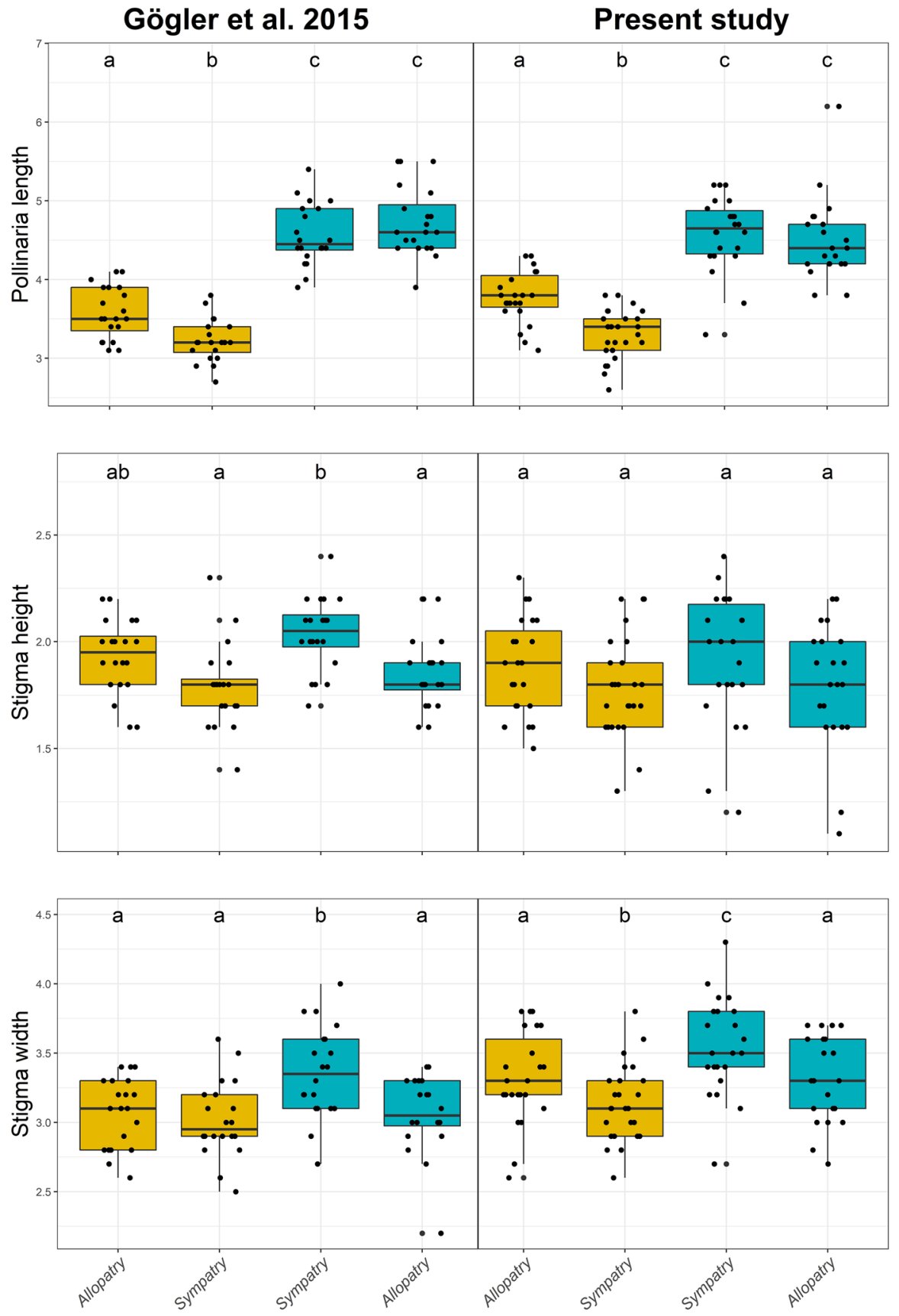

Fig. 2 Comparison among sympatric and allopatric populations of Ophrys chestermanii (yellow) and O. normanii (blue) sampled in Gögler et al. (2015) and in the present study 

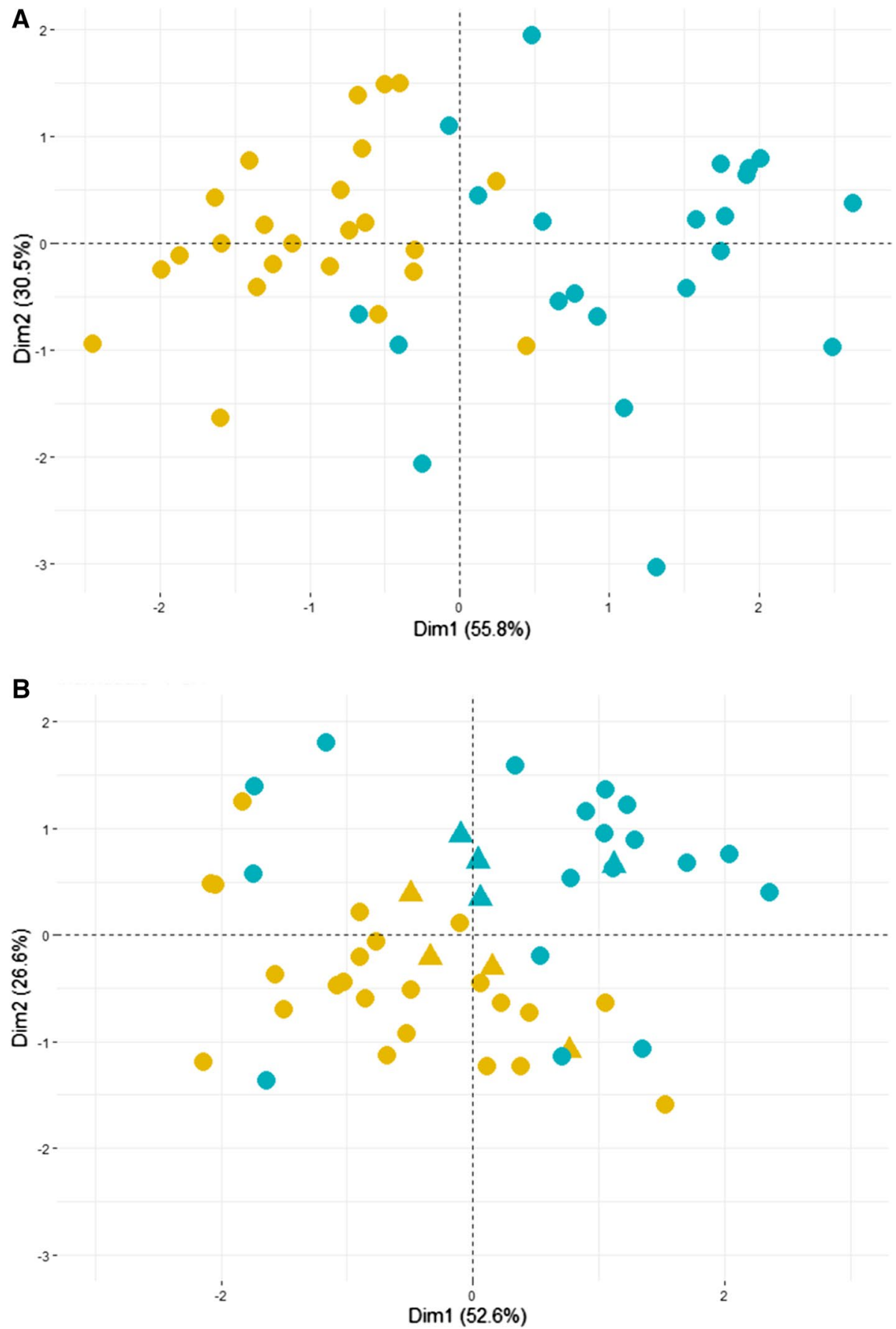

Fig. 3 Principal component analysis based on the three floral characters involved in the mechanical barrier (pollinaria length, stigma height and stigma width) of Ophrys chestermanii (yellow) and O. normanii (blue) in natural sympatry (A) and in artificial sympatry (B) in 2018. Triangles indicate individuals with hybrid fruits 
stigmatic cavity $(\mathrm{U}=20.000, \mathrm{Z}=-1.733 ; P=0.096)$ or in terms of width of stigmatic cavity $(\mathrm{U}=42.000, \mathrm{Z}=-0.143 ; P=0.918)$. Hybridizing and non-hybridizing individuals of $O$. normanii were not significantly different in terms of height of stigmatic cavity $(\mathrm{U}=27.5000, \mathrm{Z}=-0.591 ; P=0.574)$ or in terms of width of stigmatic cavity $(\mathrm{U}=18.000, \mathrm{Z}=-1.446 ; P=0.172)$.

Mean pollination success (calculated as number of fruits relative to the number of flowers) ranged from 0.31 to 0.53 in the two sampled years (Table 1). In artificial sympatry, $O$. chestermanii and $O$. normanii showed similar levels of pollination success in $2017(\mathrm{U}=264.000, \mathrm{Z}=-1.122, P=0.262, \mathrm{n}=51)$ and in $2018(\mathrm{U}=275.000$, $\mathrm{Z}=-1.718, P=0.086, \mathrm{n}=55)$. In natural sympatry, $O$. chestermanii and $O$. normanii showed similar levels of pollination success in $2017(\mathrm{U}=366.500, \mathrm{Z}=-0.196$, $P=0.844, \mathrm{n}=55)$ and in $2018(\mathrm{U}=321.000, \mathrm{Z}=-0.729, \mathrm{P}=0.466, \mathrm{n}=54)$. Putting the two species together, in 2017 the natural sympatric population showed significantly higher pollination success than artificial sympatry $(U=836.000, Z=-3.634, P<0.001$, $\mathrm{n}=106)$ and in $2018(\mathrm{U}=1146.000, \mathrm{Z}=-2.080, P<0.038, \mathrm{n}=109)$. None of the investigated floral characters was correlated to pollination success, either in $O$. chestermanii or in O. normanii (Table S1).

Hybrid fitness was lower than parental fitness in terms of fruit production after manual crosses. From the 6 artificial pollinations carried out between the three F1 artificial hybrids only one developed into a fruit (i.e., 16.6\%). Fruit production in crosses among F1 hybrids is thus significantly lower than that of intraspecific crosses reported in Gögler et al. (2015): 14 out of 15, i.e. $93.3 \%$, in $O$. normanii and 11 out of 14 , i.e. $80 \%$, in $O$. chestermanii, average fruiting production 86.65\%) (Fisher's exact test, $p=0.0021$ ). In the single fruit obtained in crosses between F1 hybrids, we observed no viable seeds.

From the two putative hybrids identified by their additive genetic profile in the newly discovered sympatric zone of Buggerru (loc. San Niccolò), we collected two fruits from natural pollinated flowers. Seed viability in these two fruits was $37.1 \%$ on average. Seed viability in these natural hybrids is thus significantly lower than that of intraspecific crosses reported in Gögler et al. (2015; $49.51 \%$ in $O$. normanii and $62.67 \%$ in $O$. chestermanii, average intraspecific crosses 50.09\%) (Fisher's exact test, $p=0.0105$ ). Thus, also in natural conditions, hybrid fitness was lower than parental fitness, in terms of seed viability.

Table 1 Pollination success (Mean and standard deviation, SD) of Ophrys chestermanii and $O$. normanii in artificial (AS) and in natural sympatry (NS)

\begin{tabular}{lll}
\hline Population & Sampling year & $\begin{array}{l}\text { Pollination success } \\
\text { Mean }(+/- \text { SD) }\end{array}$ \\
\hline Ophrys chestermanii AS & 2017 & $0.31(+/-0.26)$ \\
O. normanii AS & 2017 & $0.37(+/-0.29)$ \\
O. chestermanii AS & 2018 & $0.32(+/-0.26)$ \\
O. normanii AS & 2018 & $0.42(+/-0.24)$ \\
O. chestermanii NS & 2017 & $0.50(+/-0.18)$ \\
O. normanii NS & 2017 & $0.53(+/-0.31)$ \\
O. chestermanii NS & 2018 & $0.42(+/-0.15)$ \\
O. normanii NS & 2018 & $0.51(+/-0.31)$ \\
\hline
\end{tabular}




\section{Discussion}

Despite a long-standing debate regarding the terminology, reinforcement may be intended as the evolution of divergence in any reproductive character in sympatric plant species in response to competition, or in response to the costs associated with sharing pollen with another species (i.e., the loss of pollen to other species, the clogging of stigmas with heterospecific pollen, or the potential for unfit hybrid seed; Pfennig and Pfennig 2009; Beans 2014). Natural selection can act in sympatric populations to strengthen reproductive isolation between hybridizing species: this may occur between incipient sister species, but also between distinct species. Here, we investigated two species belonging to different Ophrys lineages (Gögler et al. 2009; Breitkopf et al. 2015), that converged in attracting the same species-specific pollinator. The two species apparently have no premating isolation with the exclusion of morphological divergence among floral characters that produces a mechanical barrier in sympatry. The floral characters potentially involved in this barrier were found to be more differentiated in sympatry than in allopatry, suggesting the presence of reproductive character displacement (Gögler et al. 2015).

While the related literature offers ample evidence and theoretical support, experimental tests validating the role of characters in RCD are less common, particularly between distant plant species, as several criteria need to be satisfied to show reinforcement (as explicitly identified in Hopkins 2013). Here, we experimentally tested the role of floral divergence in RCD by building up an artificial sympatric population with allopatric individuals and we found that in contrast to sympatric individuals, allopatric individuals widely hybridize once in contact. This finding supports the role of character displacement in generating the mechanical barrier that halts costly hybridization in these two orchids when in sympatry.

The observation of character displacement between allopatric and sympatric populations does not represent evidence for RCD, as a putative character displacement pattern could be explained by any number of other biotic and abiotic factors. A prerequisite for the evolution of RCD is that natural plant populations contain heritable variation in the displacing characters and that the difference is not (only) due to plasticity. Given the complexity of orchid life cycle and the difficulty in cultivation we cannot perform crosses for true estimates of heritability. However, we found that individuals from the same natural sympatric population sampled by Gögler et al. (2015) were not significantly different from those collected in present study and that character difference was maintained between species (with the exception of tepal length, a neutral character for pollination) (Fig. 2).

If there is a genetic basis to the difference between allopatric and sympatric individuals, then this same difference should be apparent when individuals from allopatric populations are grown together (in a sort of common garden). Accordingly, we found that character differences between populations were maintained when allopatric individuals of $O$. normanii were transferred in the $O$. chestermanii population to build the artificial sympatric zone (Fig. 1). Furthermore, by comparing character expression in the same individuals grown in allopatric and sympatric arrays, we also tested for plasticity, as the putative character displacement pattern may be caused by plastic responses to local environmental conditions. This was done across two growing years, as, in terrestrial orchids, the epigean part of the plant is renewed yearly from the small bulb-like tuber generated in the previous growing season. We found that the investigated floral characters still have a plasticity component, while maintaining the difference between allopatric and sympatric arrays across years (Fig. 2). It has been suggested that RCD may occur more readily in plastic species, because plasticity permits survival among competitors long enough for selection to narrow 
the reaction norm of each species in opposite directions (Beans 2014). However, we did not find that sympatric individuals have a more fixed sympatric phenotype than allopatric ones (Rice and Pfennig, 2007), as character variance was comparable across sites and years (Figs. 1 and 2), so that gene flow, standing genetic variation, plasticity and character complexity still subtend the actual variation, in spite of the selective pressure on divergent characters in sympatric zones.

Sympatric and allopatric sites investigated here are geographically proximate (approx. $20 \mathrm{~km}$, Fig. S2) and are very likely to have similar resource availability in terms of abundance of the specific pollinator. By comparing pollination success between natural and artificial sympatric areas, we found that it was slightly, but significantly, higher in natural sympatry (Table 1). However, in artificial sympatry, pollination success was not correlated to any of the investigated characters (Supplementary Table 1). This suggests that character variation does not affect plant pollination success by optimizing the pollinator resources (as expected for ECD). Interestingly, and as typically found in sexually deceptive orchids, the plants in all the sites were severely pollinator limited (i.e. a very low number of fruits compared to flower number), a condition that boosts the action of pollinator mediated selection.

A prerequisite for the existence of reproductive character displacement is that mating between diverging taxa and/or hybridization is costly. Gögler et al. (2015) found that artificial F1 hybrids can grow healthily and bloom, thus excluding early postzygotic barriers but without testing any late postzygotic barrier. Here we found that crosses between the same artificial F1 hybrids result in low fruit production and no viable seeds (i.e. late postzygotic barrier). In addition, fruits of the natural hybrids identified through a molecular approach in Buggerru (loc. San Niccolò), contained a lower amount of viable seeds when compared to parental species in same conditions. Late generation hybrids can partially restore fertility, particularly when fecundated by pollen of parental species (i.e. backcrosses). Although our approach impedes the identification of both parental individuals (i.e. the identification of pollen donor), we found that seed viability is still highly reduced when the hybrid is the ovule donor.

Increased production of maladapted hybrid progeny in artificial sympatry compared to a natural sympatric area, where character displacement occurred, confirms that the decrease in interspecific mating increases net fitness of individuals with displaced characters, and that natural selection favours divergence in phenotypes among sympatric species. The allopatric morphs produced 75\% and 25\% hybrid fruits in 2017 and 2018, while the sympatric morphs produced no hybrid fruits in examined years, indicating that the sympatric morphs had probably evolved to almost completely halt interspecific pollination. This is supported by the fact that no hybrid genotype was detected in the molecular analysis of Gögler et al. (2009) and that only two putative hybrid plantlets were identified in the subsequent analysis of Gögler et al. (2015) in the natural sympatric zone of Perdu Carta. On a cautionary note, however, it must be noted that the high level of interspecific pollination detected in the artificial sympatry could be partially due to the lower average distance between individuals of the two species (around $10 \mathrm{mt}$ ) compared to the species distribution in the natural sympatric area (around $50 \mathrm{mt}$ ). Shorter distance between the two species in the artificial sympatric array may have favored pollinator flights between species and thus the high interspecific pollen transfer (50\% on average, i.e., as expected from random chance). We suspect that the incomplete admixture of the two species on a small scale in the natural sympatric zone allows for the potential contribution of microhabitat isolation in further reducing interspecific pollen flow.

However, in contrast to the expectation that individuals with similar phenotypes should interact more strongly, by comparing the plant phenotype and the seed progeny we found 
that hybridizing and non-hybridizing individuals of $O$. chestermanii and of $O$. normanii were not significantly different in terms of height of stigmatic cavity or in terms of width of stigmatic cavity (i.e. the displaced characters), so that we do not detect a direct link between the displaced character and decrease hybridization or mating between diverging taxa (Fig. 3). In sympatry, the main factor preventing gene flow between these two sympatric species is a combination of the longer pollinaria of $O$. normanii and the smaller and differently shaped stigmatic cavity in $O$. chestermanii, a sort of lock and key mechanism as clearly confirmed by video recording (Gögler et al. 2015). We argue that this is an intrinsic limitation of our experimental design as, by analyzing fruits, we can only measure the displaced characters in the ovule donor plant, but not in the pollen donor plant, so that we cannot fully evaluate the effect of the characters combination.

Our study on these rare and endemic orchids has evident limits in the replications of allopatric and sympatric zones and of in the small number of examined artificial and natural hybrids. Still, our results clearly indicate that the character displacement detected in the area of secondary contact can be considered proof of reinforcing selection against maladaptive hybridization (e.g., RCD), rather than ECD resulting from competition for pollinator service (see above). Differently from generalist species, increased floral divergence in highly specialized plants, which eventually come into secondary contact and share their specialized pollinator, is expected to be more strongly constrained by the specific requirements of the pollinator. Indeed, both the odor bouquet and phenology are not displaced between species in sympatry, as they may potentially have detrimental effects on plant pollination success (Smith and Rausher 2008; Gögler et al. 2015).This, coupled with a condition of strong pollinator limitation, as typical of deceptive orchids, suggests that the reinforcing pressure can be very strong, so that we detected phenotypic divergence between sympatric and allopatric populations, even in a very narrow geographic context (less than $100 \mathrm{~km}^{2}$ ) and, most likely, in the presence of intraspecific gene flow between allopatric and sympatric populations.

Compared to generalist pollinated plants that may vary in reinforcing processes to avoid overlap in pollinators in contact zones (as shifting in phenology, changes in pollinator spectrum, scent, colour, etc.) highly specialist pollinated plants are expected to be severely constrained by the very strict interaction with their unique pollinator. Nevertheless, here we report on a case of reinforcement of reproductive isolation upon secondary contact occurring, in plants, like orchids, with extremely specialized pollination systems.

Supplementary Information The online version contains supplementary material available at https://doi. org/10.1007/s10682-021-10149-2.

Author contributions S.C. and M.A. conceived and designed the study; P.C. and G.S. performed the experiments; G.S. analysed the data; M.R.B.L. performed morphological measurements; D.C. performed molecular analysis; S.C., M.A. and G.S. wrote a first draft of the manuscript. All authors contributed to the writing.

Funding The research was supported by University internal grant to MA and SC.

\section{Declarations}

Conflict of interest The authors declare that they have no competing interests.

Consent to participate All authors have given their consensus to participate.

Consent for publication All authors have given their consensus for publication.

Data availability All data will be archived on Dryad upon acceptance. 
Open Access This article is licensed under a Creative Commons Attribution 4.0 International License, which permits use, sharing, adaptation, distribution and reproduction in any medium or format, as long as you give appropriate credit to the original author(s) and the source, provide a link to the Creative Commons licence, and indicate if changes were made. The images or other third party material in this article are included in the article's Creative Commons licence, unless indicated otherwise in a credit line to the material. If material is not included in the article's Creative Commons licence and your intended use is not permitted by statutory regulation or exceeds the permitted use, you will need to obtain permission directly from the copyright holder. To view a copy of this licence, visit http://creativecommons.org/licenses/by/4.0/.

\section{References}

Aizen MA, Vázquez DP (2006) Flowering phenologies of hummingbird plants from the temperate forest of southern South America: is there evidence of competitive displacement? Ecography 29:357-366

Armbruster WS, Herzig AL (1984) Partitioning and sharing of pollinators by four sympatric species of Dalechampia (Euphorbiaceae) in Panama. Ann Mo Bot Gard 71:1-16

Armbruster WS, Muchhala N (2009) Associations between floral specialization and species diversity: cause, effect, or correlation? Evol Ecol 23:159

Ayasse M, Stökl J, Francke W (2011) Chemical ecology and pollinator-driven speciation in sexually deceptive orchids. Phytochemistry 72:1667-1677

Bateman RM, Sramkó G, Paun O (2018) Integrating restriction site-associated DNA sequencing (RAD-seq) with morphological cladistic analysis clarifies evolutionary relationships among major species groups of bee orchids. Ann Bot 121:85-105

Beans CM (2014) The case for character displacement in plants. Ecol Evol 4:862-875

Bell JM, Karron JD, Mitchell RJ (2005) Interspecific competition for pollination lowers seed production and outcrossing in Mimulus ringens. Ecology 86:762-771

Breitkopf H, Onstein RE, Cafasso D, Schlüter PM, Cozzolino S (2015) Multiple shifts to different pollinators fuelled rapid diversification in sexually deceptive Ophrys orchids. New Phytol 207:377-389

Brown WL, Wilson EO (1956) Character displacement. Syst Zool 5:49-64

Caruso CM (2000) Competition for pollination influences selection on floral traits of Ipomopsis aggregata. Evolution 54:1546-1557

Coyne JA, Orr HA (2004) Speciation. Sinauer Associates, Sunderland, MA

Dayan T, Simberloff D (2005) Ecological and community-wide character displacement: the next generation. Ecol Lett 8:875-894

Dobzhansky T (1940) Speciation as a stage in evolutionary divergence. Am Nat 74:312-321

Doyle JJ, Doyle JL (1987) A rapid DNA isolation procedure for small quan- tities of fresh leaf material. Phytochemical Bulletin. Botanical Society of America 19:11-15

Fishman L, Wyatt R (1999) Pollinator-mediated competition, reproductive character displacement, and the evolution of selfing in Arenaria uniflora (Caryophyllaceae). Evolution 53:1723-1733

Gerhardt HC (1994) Reproductive character displacement of female mate choice in the grey treefrog, Hyla chrysoscelis. Anim Behav 47:959-969

Geyer LB, Palumbi SR (2003) Reproductive character displacement and the genetics of gamete recognition in tropical sea urchins. Evolution 57:1049-1060

Gögler J, Stökl J, Sramkova A, Twele R, Francke W, Cozzolino S, Cortis P, Scrugli A, Ayasse M (2009) Ménage À Trois-Two Endemic species of deceptive orchids and one pollinator species. Evolution 63:2222-2234

Gögler J, Stökl J, Cortis P, Beyrle H, Barone Lumaga MR, Cozzolino S, Ayasse M (2015) Increased divergence in floral morphology strongly reduces gene flow in sympatric sexually deceptive orchids with the same pollinator. Evol Ecol 29:703-717

Haavie J, Borge T, Bures S, Garamszegi LZ, Lampe HM, Moreno J, Qvarnström A, Török J, Sætre GP (2004) Flycatcher song in allopatry and sympatry-convergence, divergence and reinforcement. J Evol Biol 17:227-237

Hopkins R (2013) Reinforcement in plants. New Phytol 197:1095-1103

Hopkins R, Rausher MD (2012) Pollinator-mediated selection on flower color allele drives reinforcement. Science 335:1090-1092

Howard DJ (1993) Reinforcement: origin, dynamics, and fate of an evolutionary hypothesis. Hybrid Zones Evol Process 46-69

Kozak KH, Wiens J (2006) Does niche conservatism promote speciation? a case study in North American salamanders. Evolution 60:2604-2621 
Lemmon EM (2009) Diversification of conspecific signals in sympatry: geographic overlap drives multidimensional reproductive character displacement in frogs. Evolution 63:1155-1170

Lussu M, De Agostini A, Marignani M, Cogoni A, Cortis P (2018) Ophrys annae and Ophrys chestermanii: an impossible love between two orchid sister species. Nord J Bot 36:e01798

Lussu M, De Agostini A, Cogoni A, Marignani M, Cortis P (2019) Does size really matter? a comparative study on floral traits in orchids with two different pollination strategies. Plant Biol 21:961-966

Martin MM, Harding J (1981) Evidence for the evolution of competition between two species of annual plants. Evolution 35:975-987

Mitchell RJ, Irwin RE, Flanagan RJ, Karron JD (2009) Ecology and evolution of plant-pollinator interactions. Ann Bot 103:1355-1363

Muchhala N, Potts MD (2007) Character displacement among bat-pollinated flowers of the genus Burmeistera: analysis of mechanism, process and pattern. Proc R Soc B 274:2731-2737

Noor MA (1995) Speciation driven by natural selection in Drosophila. Nature 375:674

Noor MA (1999) Reinforcement and other consequences of sympatry. Heredity 83:503-508

Norton NA, Fernando MTR, Herlihy CR, Busch JW (2015) Reproductive character displacement shapes a spatially structured petal color polymorphism in Leavenworthia stylosa. Evolution 69:1191-1207

Pfennig K, Pfennig D (2009) Character displacement: ecological and reproductive responses to a common evolutionary problem. Q Rev Biol 84:253-276

Price TD (2007) Speciation in birds. Roberts \& Company, Greenwood Village, Colorado

Rice AM, Pfennig DW (2007) Character displacement: in situ evolution of novel phenotypes or sorting of pre-existing variation? J Evol Biol 20:448-459

Roda F, Mendes FK, Hahn MW, Hopkins R (2017) Genomic evidence of gene flow during reinforcement in Texas Phlox. Mol Ecol 26:2317-2330

Rundle HD, Schluter D (1998) Reinforcement of stickleback mate preferences: sympatry breeds contempt. Evolution 52:200-208

Scopece G, Musacchio A, Widmer A, Cozzolino S (2007) Patterns of reproductive isolation in Mediterranean deceptive orchids. Evolution 61:2623-2642

Scrugli A (1990) Orchidee spontanee della sardegna origine, morfologia, riproduzione, impollinazione, ibridazione, anomalie, fioritura. Edizioni Della Torre, Cagliari, Italy

Servedio MR, Noor MA (2003) The role of reinforcement in speciation: theory and data. Ann Rev Ecol Evol Syst 34:339-364

Smith RA, Rausher MD (2008) Selection for character displacement is constrained by the genetic architecture of floral traits in the ivyleaf morning glory. Evolution 62:2829-2841

Sobel JM, Chen GF, Watt LR, Schemske DW (2010) The biology of speciation. Evolution 64:295-315

Spriggs EL, Schlutius C, Eaton DA, Park B, Sweeney PW, Edwards EJ, Donoghue MJ (2019) Differences in flowering time maintain species boundaries in a continental radiation of Viburnum. Am J Bot 106:833-849

Stone GN, Willmer P, Rowe JA (1998) Partitioning of pollinators during flowering in an African Acacia community. Ecology 79:2808-2827

Todesco M, Pascual MA, Owens GL, Ostevik KL, Moyers BT, Hübner S, Heredia SM, Hahn MA, Caseys C, Bock DG, Rieseberg LH (2016) Hybridization and extinction. Evol Appl 9:892-908

Van der Niet T, Johnson SD, Linder HP (2006) Macroevolutionary data suggest a role for reinforcement in pollination system shifts. Evolution 60:1596-1601

Waser NM (1978) Interspecific pollen transfer and competition between co-occurring plant species. Oecologia 36:223-236

Whitehead MR, Peakall R (2014) Pollinator specificity drives strong prepollination reproductive isolation in sympatric sexually deceptive orchids. Evolution 68:1561-1575

Widmer A, Lexer C, Cozzolino S (2009) Evolution of reproductive isolation in plants. Heredity 102:31-38

Xu SQ, Schluter PM, Scopece G, Breitkopf H, Gross K, Cozzolino S, Schiestl FP (2011) Floral isolation is the main reproductive barrier among closely related sexually deceptive orchids. Evolution 65:2606-2620

Zitari A, Scopece G, Helal AN, Widmer A, Cozzolino S (2012) Is floral divergence sufficient to maintain species boundaries upon secondary contact in Mediterranean food-deceptive orchids? Heredity 108:219-228

Publisher's Note Springer Nature remains neutral with regard to jurisdictional claims in published maps and institutional affiliations. 